\title{
Multiplicative point process as a model of trading activity
}

\author{
V. Gontis and B. Kaulakys ${ }^{1}$ \\ Institute of Theoretical Physics and Astronomy, Vilnius University \\ A.Goštauto 12, LT-01108 Vilnius, Lithuania \\ gontis@ktl.mii.lt
}

\begin{abstract}
Signals consisting of a sequence of pulses show that inherent origin of the $1 / f$ noise is a Brownian fluctuation of the average interevent time between subsequent pulses of the pulse sequence. In this paper we generalize the model of interevent time to reproduce a variety of self-affine time series exhibiting power spectral density $S(f)$ scaling as a power of the frequency $f$. Furthermore, we analyze the relation between the power-law correlations and the origin of the power-law probability distribution of the signal intensity. We introduce a stochastic multiplicative model for the time intervals between point events and analyze the statistical properties of the signal analytically and numerically. Such model system exhibits power-law spectral density $S(f) \sim 1 / f^{\beta}$ for various values of $\beta$, including $\beta=1 / 2,1$ and $3 / 2$. Explicit expressions for the power spectra in the low frequency limit and for the distribution density of the interevent time are obtained. The counting statistics of the events is analyzed analytically and numerically, as well. The specific interest of our analysis is related with the financial markets, where long-range correlations of price fluctuations largely depend on the number of transactions. We analyze the spectral density and counting statistics of the number of transactions. The model reproduces spectral properties of the real markets and explains the mechanism of power-law distribution of trading activity. The study provides evidence that the statistical properties of the financial markets are enclosed in the statistics of the time interval between trades. A multiplicative point process serves as a consistent model generating this statistics.
\end{abstract}

Keywords: Stochastic processes; Econophysics; Financial markets; Point processes; $1 / f$ noise

PACS: 05.40.-a; 02.50.Ey; 89.65.Gh

\section{Introduction}

Complex collective phenomena usually are responsible for the power-laws which are universal and independent of the microscopic details of the phenomenon. Examples in physics are numerous. Power-laws are intrinsic features of the economic and financial data, as well. The aim of this contribution is to analyze a relationship between the origin of the power-law distribution and the power-law correlations in financial time series.

There are numerous studies of power-law probability distributions in various economic systems [1-15]. The key result in recent findings is that the cumulative distributions of returns and trading activity can be well described by a power-law

\footnotetext{
${ }^{1}$ Corresponding author. Tel. +370-5-2620859; fax +370-5-2125361.

E-mail address: kaulakys@itpa.lt (B. Kaulakys)
} 
asymptotic behavior, characterized by an exponent $\lambda \approx 3$, well outside the stable Levy regime $0<\lambda<2[13-15]$. Empirical studies confirm the power-law asymptotic behavior with stable exponent for different time scales. Since these empirical distributions are neither Levy stable nor invariant under addition, new stochastic models with long range correlations and power-law asymptotic behavior are of great interest [14].

The random multiplicative process built into the model of wealth distribution yields Pareto power-law [2]. The generalized Lotka-Volterra dynamics is in the use for various systems including financial markets [9]. However, these models generically lead to non-universal exponents and do not explain the power-law autocorrelations and observable spectral density of financial time series [11]. The time-correlations in the financial time series are studied extensively as well [13-17]. Recent investigations [13-16] provide empirical evidence that the long-range correlations for volatility are due to the trading activity, measured by a number of transactions $N$. Therefore, various stochastic models of trading activity or for waiting time between transactions have to be analysed for reproduction the statistics of volatility in financial markets.

Recently we tried to adapt the model of $1 / f$ noise based on the Brownian motion of time interval between subsequent pulses, proposed in [18-21], to model the share volume traded in the financial markets [22]. The idea to transfer long time correlations into the stochastic process of the time interval between trades or time series of trading activity is in consistence with the detailed studies of the empirical financial data $[13,16]$ and can reproduce the spectral properties of the financial time series [22]. However, the investigation of the model revealed, that the simple additive Brownian model of time interval between trades failed to reproduce the power-law probability density function (pdf) of the trading activity [23].

On the other hand, several authors have shown empirically that the fluctuations of various financial time series possess multifractal statistics [24-28]. Therefore, in this paper we introduce the stochastic multiplicative model for the time interval between trades and analyze the statistical properties of the trading activity analytically and numerically. The model is in consistence with the results of statistical analysis of the empirical financial time series and reveals the relationship between the power-law probability distribution and the power-law spectral density of the financial time series. Moreover, the analytically solvable model [18-21] results only in exact $1 / f$ noise while in the papers $[22,23]$ essentially only numerical analysis has been undertaken. Here we generalize the model defining the time series exhibiting the power spectral density $1 / f^{\beta}$ with $0.5 \lesssim \beta \lesssim 1.5$ and the power-law probability density of the trading activity. Explicit analytical expressions for the power spectra and for pdf of the trading activity $N$ are obtained and analysed.

\section{The model}

We consider a signal $I(t)$ as a sequence of the random correlated pulses

$$
I(t)=\sum_{k} a_{k} \delta\left(t-t_{k}\right)
$$

where $a_{k}$ is a contribution to the signal of one pulse at the time moment $t_{k}$, e.g., a contribution of one transaction to the financial data. Signal (11) represents a point process used in a large variety of systems with the flow of point objects or subsequent actions. When $a_{k}=\bar{a}$ is constant, the point process is completely described by 
the set of times of the events $\left\{t_{k}\right\}$ or equivalently by the set of interevent intervals $\left\{\tau_{k}=t_{k+1}-t_{k}\right\}$. Various stochastic models of $\tau_{k}$ can be introduced to define a stochastic point process. In papers [18-21] it has been shown analytically that the relatively slow Brownian fluctuations of the interevent time $\tau_{k}$ yield $1 / f$ fluctuations of the signal (11).

Power spectral density of the signal $I(t)$ is

$$
\begin{aligned}
S(f) & =\lim _{T \rightarrow \infty}\left\langle\frac{2 \bar{a}^{2}}{T}\left|\int_{t_{i}}^{t_{f}} I(t) \exp (-i 2 \pi f t) d t\right|^{2}\right\rangle \\
& =\lim _{T \rightarrow \infty}\left\langle\frac{2 \bar{a}^{2}}{T} \sum_{k=k_{\min }}^{k_{\max }} \sum_{q=k_{\min }-k}^{k_{\max }-k} \exp (-i 2 \pi f \Delta(k ; q))\right\rangle
\end{aligned}
$$

where the brackets $\langle\ldots\rangle$ denote the averaging over the realizations of the process, $T=t_{f}-t_{i}$ is the observation time, $\Delta(k ; q)=t_{k+q}-t_{k}$ is the time difference between the pulses occurrence times $t_{k+q}$ and $t_{k}$, while $k_{\min }$ and $k_{\max }$ are minimal and maximal values of the index $k$ in the interval of observation $T$.

We adapt and generalize the model of $1 / f$ noise previously proposed in $[18-21]$ to model the share volume traded in the financial markets $[13,16]$. It is useful to define a discrete time series with equal time intervals $\tau_{d}$. Integration of the signal $I(t)$ over subsequent time intervals of length $\tau_{d}$ results in a discrete time series and, by analogy with financial time series, we will call it the volume $V_{j}$,

$$
V_{j}=\int_{t_{j}}^{t_{j}+\tau_{d}} I(t) d t=\sum_{t_{j}<t_{k}<t_{j}+\tau_{d}} a_{k}, \quad t_{j}=j \tau_{d} .
$$

The number of trades $N_{j}$ in the time interval $\left[t_{j}, t_{j}+\tau_{d}\right]$ is defined by the same equation (3) with $a_{k} \equiv 1$ and $N_{j} \equiv V_{j}$. Then the power spectral density $S\left(f_{s}\right)$ of the discrete signal $N_{j}$ may be calculated by the FFT as

$$
S\left(f_{s}\right)=\left\langle\frac{2}{\tau_{d} n}\left|\sum_{j=1}^{n} N_{j} \exp (-i 2 \pi(s-1)(j-1))\right|^{2}\right\rangle .
$$

Here the discrete frequencies $f_{s}=(s-1) / T, s=1,2, \ldots n$ and $T=\tau_{d} n$. For frequencies $f_{s} \ll 1 / \tau_{d}$ the power spectral density defined by equation (4) coincides with that given by equation (2).

In this paper we investigate the statistical properties of the time series $N_{j}$, when the sequence of interevent time $\tau_{k}$ is generated by a multiplicative stochastic process. First of all the multiplicativity is an essential feature of processes in economics $[1,7]$. Multiplicative stochastic processes yield multifractal intermittency and are able to produce power-law probability distribution functions. We base our study on the generic multiplicative process for the interevent times, written as

$$
\tau_{k+1}=\tau_{k}+\gamma \tau_{k}^{2 \mu-1}+\tau_{k}^{\mu} \sigma \varepsilon_{k} .
$$

Here the interevent time $\tau_{k}$ fluctuates due to the external random perturbation by a sequence of uncorrelated normally distributed random variable $\left\{\varepsilon_{k}\right\}$ with zero expectation and unit variance, $\sigma$ denotes the standard deviation of the white noise and $\gamma \ll 1$ is a damping constant. 
We will restrict the diffusion of the interevent time according to equation (5) to the finite interval $\left[\tau_{\min }, \tau_{\max }\right]$, i.e., $0<\tau_{\min }<\tau_{k}<\tau_{\max }$. The most simple case is the pure multiplicativity of $\tau_{k}$, i.e., when $\mu=1$. Other values of $\mu$ reproduce the power-laws as well and explicit expressions can be derived without the loss of generality.

The iterative relation (5) can be rewritten as Langevine stochastic differential equation in $k$-space.

$$
\frac{d \tau_{k}}{d k}=\gamma \tau_{k}^{2 \mu-1}+\tau_{k}^{\mu} \sigma \xi(k)
$$

Here we interpret $k$ as continuous variable while

$$
\left\langle\xi(k) \xi\left(k^{\prime}\right)\right\rangle=\delta\left(k-k^{\prime}\right) .
$$

The steady state solution of the corresponding stationary Fokker-Planck equation with zero flow gives the probability density function for $\tau_{k}$ in the $k$-space (see, e.g., [29])

$$
P_{k}\left(\tau_{k}\right)=C \tau_{k}^{\alpha}, \quad \alpha=2 \gamma / \sigma^{2}-2 \mu
$$

and $C$ has to be defined from the normalization

$$
\int_{\tau_{\min }}^{\tau_{\max }} P_{k}(\tau) d \tau=1
$$

The solution (7) assumes Ito convention involved in the relation between expressions (5), (6) and (7).

As the probability distribution function (7) follows a power-law, we expect that this would result in the power-law behavior in other statistics, as well. In the next Section we will obtain the power spectral density defined by equation (2).

\section{Power spectral density}

The power spectral density is a well-established measure of long time correlations and is widely used in stochastic systems. As it has been already shown [18-21] the point process with the Brownian interevent time exhibits $1 / f$ noise.

We will derive the formula for the power spectral density of the multiplicative stochastic point process model, defined by equations (5) and (6) for the interevent time.

Let us rewrite equation (2) as [21]

$$
S(f)=\lim _{T \rightarrow \infty} \frac{2 \bar{a}^{2}}{T} \sum_{k, q} \chi_{\Delta(k: q)}(2 \pi f)
$$

where $\chi_{\Delta(k: q)}(2 \pi f)$ is the characteristic function for the probability distribution of $\Delta(k ; q)$. For the normal distribution of $\Delta(k ; q)$ formula (8) takes the form

$$
S(f)=\lim _{T \rightarrow \infty} \frac{2 \bar{a}^{2}}{T} \sum_{k, q} \exp \left\{i 2 \pi f\langle\Delta(k ; q)\rangle-2 \pi^{2} f^{2} \sigma_{\Delta}^{2}(k ; q)\right\}
$$

where $\sigma_{\Delta}^{2}$ is the variance of $\Delta(k ; q)$, the time difference between the pulses occurrence times $t_{k+q}$ and $t_{k}$. For $\mu=1$ the time difference $\Delta(k ; q)$ may be expressed from the solution of multiplicative stochastic equation (6) as

$$
\Delta(k ; q)=\sum_{l=1}^{q} \tau_{k} \exp \left\{\left(\gamma-\frac{1}{2} \sigma^{2}\right) l+\sigma \sum_{j=1}^{l} \varepsilon_{j}\right\} .
$$


Averaging over the normal distributions of uncorrelated $\varepsilon_{j}$ results in explicit expressions for the mean $\langle\Delta(k ; q)\rangle$ and variance $\sigma_{\Delta}^{2}(k ; q)$.

In general, for any $\mu$, the perturbative solution of equation (6] ) yields

$$
\begin{aligned}
\langle\Delta(k ; q)\rangle & =\tau_{k} q+\frac{\gamma}{2} \tau_{k}^{2 \mu-1} q^{2}+o\left(\gamma^{2}\right), \\
\sigma_{\Delta}^{2}(k ; q) & =\tau_{k}^{2 \mu} \frac{\sigma^{2}}{3} q^{3} .
\end{aligned}
$$

In the low frequency limit $f \ll \tau_{k}^{-1}$ we can replace the summation over $k$ and $q$ by the integration and take into account only the first order terms of equation (11) in the expression for the power spectral density (9). This yields

$$
\begin{gathered}
S_{\mu}(f)=\frac{4 C \bar{a}^{2}}{\bar{\tau}} \operatorname{Re} \int_{\tau_{\min }}^{\tau_{\max }} d \tau \tau^{\alpha} \int_{0}^{\infty} \exp \left\{i 2 \pi f\left(\tau q+\frac{\gamma}{2} \tau^{2 \mu-1} q^{2}\right)\right\} d q, \\
=\frac{2 C \bar{a}^{2}}{\sqrt{\pi} \bar{\tau}(3-2 \mu) f}\left(\frac{\gamma}{\pi f}\right)^{\frac{\alpha}{3-2 \mu}} I_{\operatorname{erf}}\left(x_{\min }, x_{\max }\right), \\
I_{\text {erf }}\left(x_{\min }, x_{\max }\right)=\operatorname{Re} \int_{x_{\min }}^{x_{\max }} \exp \left\{-i\left(x-\frac{\pi}{4}\right)\right\} \operatorname{erfc}(\sqrt{-i x}) x^{\frac{\alpha}{3-2 \mu}-\frac{1}{2}} d x
\end{gathered}
$$

where

$$
\bar{\tau}=\left\langle\tau_{k}\right\rangle=\frac{T}{k_{\max }-k_{\min }}
$$

is the expectation of $\tau_{k}$. Here we introduce the scaled variable $x=\frac{\pi f}{\gamma} \tau^{3-2 \mu}$ and

$$
x_{\min }=\frac{\pi f}{\gamma} \tau_{\min }^{3-2 \mu}, \quad x_{\max }=\frac{\pi f}{\gamma} \tau_{\max }^{3-2 \mu} .
$$

Expression of the power spectral density (12) is appropriate for the numerical calculations of the generalized multiplicative point process defined by equations (11) and (5). In the limit $\tau_{\min } \rightarrow 0$ and $\tau_{\max } \rightarrow \infty$ we obtain an explicit expression for $S_{\mu}(f)$

$$
S_{\mu}(f)=\frac{C \bar{a}^{2}}{\sqrt{\pi} \bar{\tau}(3-2 \mu) f}\left(\frac{\gamma}{\pi f}\right)^{\frac{\alpha}{3-2 \mu}} \frac{\Gamma\left(\frac{1}{2}+\frac{\alpha}{3-2 \mu}\right)}{\cos \left(\frac{\pi \alpha}{2(3-2 \mu)}\right)} .
$$

Equation (14) reveals that the multiplicative point process (15) results in the power spectral density $S(f) \sim 1 / f^{\beta}$ with the scaling exponent

$$
\beta=1+\frac{2 \gamma / \sigma^{2}-2 \mu}{3-2 \mu}
$$

Let us compare our analytical results (12), (13) and (14) with the numerical calculations of the power spectral density according to equations (2) and (5). In Fig. 1]we present the numerically calculated power spectral density $S(f)$ of the signal $I(t)$ for $\mu=1$ and $\alpha=2 \gamma / \sigma^{2}-2=0,-0.4$ and +0.5 . Numerical results confirm that the multiplicative point process exhibits the power spectral density scaled as $S(f) \sim 1 / f^{\beta}$. Equation (12) describes the model power spectral density very well in a wide range of parameters. The explicit formula (14) gives a good approximation of power spectral density for the parameters when $\beta \simeq 1$. In Fig. 2 we present numerical 

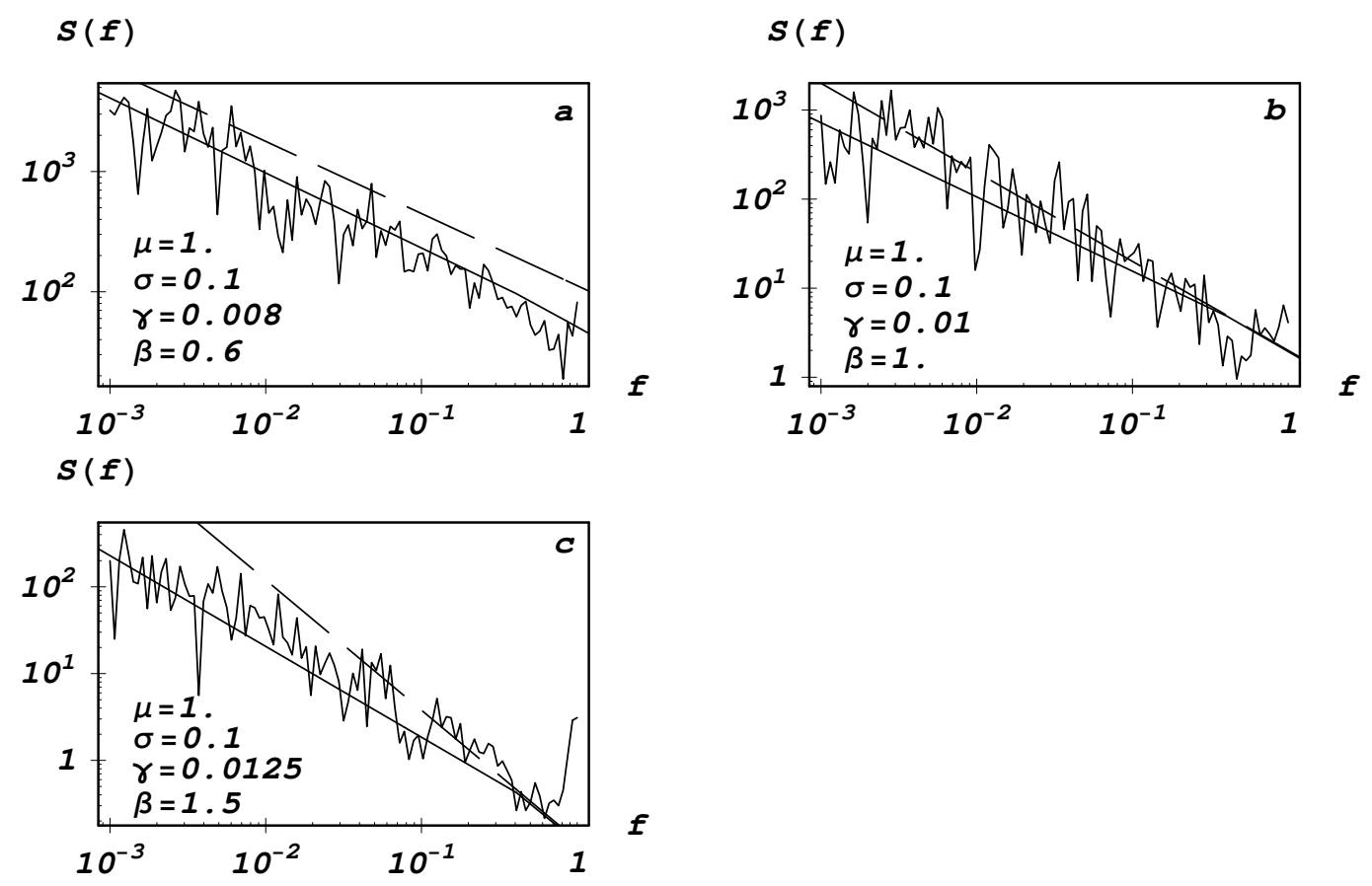

Figure 1: The power spectral density $S(f)$ of the signal $I(t)$ versus frequency $f$ calculated from the model described by equations (11), (2) and (5) with $\mu=1$. The sinous curves represent the results of the numerical simulations averaged over 5 realizations of 100000 impulse sequences with $\sigma=0.1$, the straight lines represent the analytical approximation given by integral formula (12) with $\tau_{\min }=10^{-6} ; \tau_{\max }=1$ and the dashed lines show results of explicit formula (14). (a) $\gamma=0.008$, (b) $\gamma=0.01$ and (c) $\gamma=0.0125$.

results of the power spectral density calculated for the multiplicative point process with $\mu=0.5$. In this case analytical expressions (12), (13) and (14) describe power spectral density very well in a wide range of the parameter $\gamma$. These results confirm the earlier finding [18-21] that the power spectral density is related to the probability distribution of the interevent time $\tau_{k}$ and $1 / f$ noise occurs when this distribution is flat, i.e., when $\alpha=0$.

It is likely that such a stochastic model with parameters in the region $0.5 \leq \beta \leq$ 1.5 may be adaptable for a wide variety of different systems. In this paper we will investigate applicability of the model for the financial market.

\section{Power-law probability distribution function}

The statistics of the trading activity $N$, i.e., the number of trades per time interval $\tau_{d}$ is the major task of this paper. The consistency of any model of financial markets depends on its ability to reproduce empirically measured probability distribution of the number of trades $N$, defined by equation (3). Intuitively, the pdf of $N$ is related with pdf of the rate $\nu=1 / \tau$ and with pdf of $\tau$. The pdf of $\tau_{k}$ in $k$-space is given by expression (7). It is obvious that in the actual time $t$ the pdf of $\tau$ may be written as

$$
P_{t}(\tau)=P_{k}(\tau) \tau / \bar{\tau}=C^{\prime} \tau^{\alpha+1}
$$



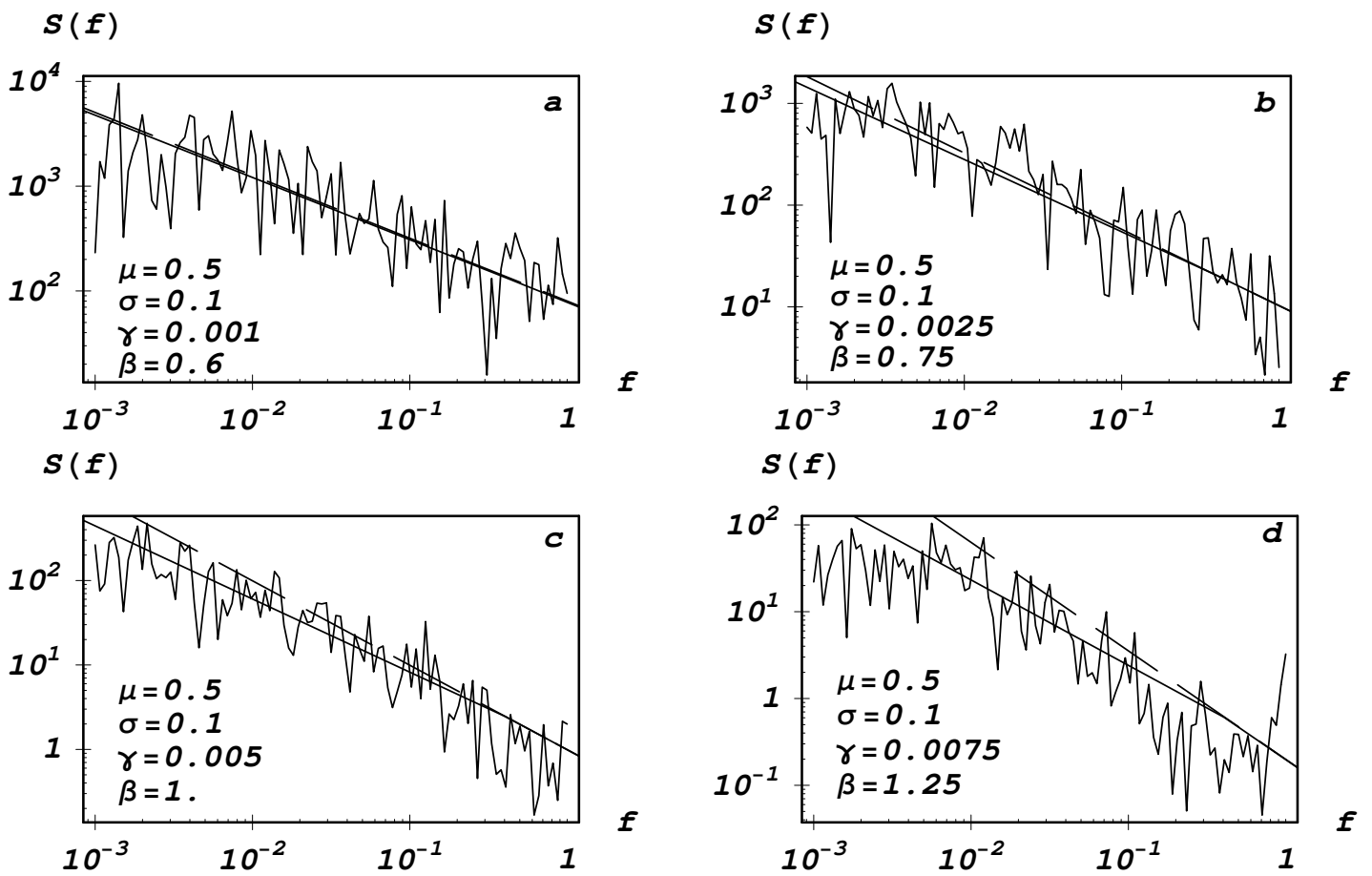

Figure 2: The same as in Fig. 1 but for $\mu=0.5$. (a) $\gamma=0.001$, (b) $\gamma=0.0025$, (c) $\gamma=0.005$ and $(\mathrm{d}) \gamma=0.0075$.

where $C^{\prime}=C / \bar{\tau}$ is a new normalization constant.

For the pure multiplicative model with $\mu=1$ equations (10) and (11) define a relationship between $N$ and $\tau$ after the substitution $\langle\Delta(k ; q)\rangle \rightarrow \tau_{d}, \tau_{k} \rightarrow \tau$ and $q \rightarrow N$, i.e.,

$$
\tau_{d}=\tau N+\frac{\gamma}{2} \tau N^{2}
$$

This relationship may be used for definition of pdf $P(N)$ from the relation $P(N) d N=P_{t}(\tau) d \tau$. We have

$$
P(N)=\frac{C^{\prime} \tau_{d}^{2+\alpha}(1+\gamma N)}{N^{3+\alpha}\left(1+\frac{\gamma}{2} N\right)^{3+\alpha}}
$$

This equation yields limiting cumulative distributions for $N$,

$$
P_{>}(N)=\int_{N}^{+\infty} P(N) d N \sim \begin{cases}\frac{1}{N^{2+\alpha}}, & N \ll \gamma^{-1}, \\ \frac{1}{N^{4+2 \alpha}}, & N \gg \gamma^{-1} .\end{cases}
$$

Let us compare probability distribution function for $N$ obtained from the numerical simulation of the model with the analytical results (18) and (19). It is convenient to use the amplitude versus rank plot (see, e.g., [30] ) for definition of the exponent $\lambda$ of the cumulative distribution $P_{>}(N) \sim 1 / N^{\lambda}$. We plot the value of the $n$-th rank variable $N(n)$ as a function of its rank $n$. If $P_{>}(N)$ behaves asymptotically when $n \rightarrow 0$ as a power-law, one gets a straight line in log-log coordinates of $N$ and $n$, with the slope equal to $-1 / \lambda$. In Fig. 3 we present the plot for $N(n)$ calculated numerically for pure multiplicative model $(\mu=1)$ with the parameters yielding the best fit to the empirical data. 


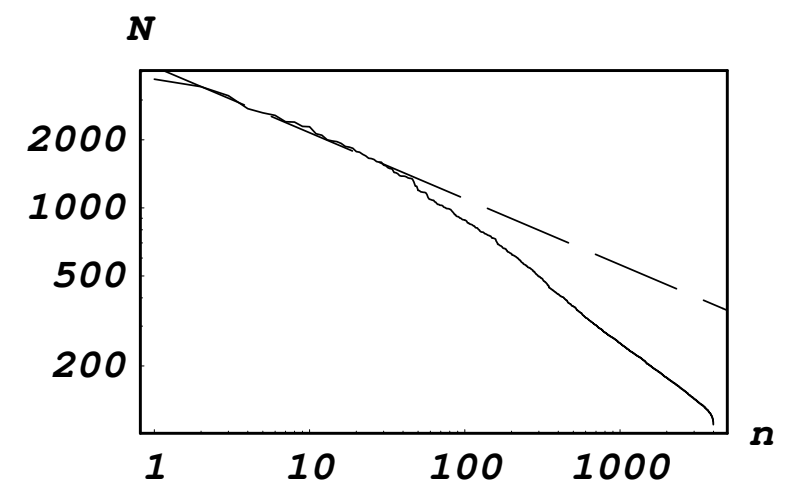

Figure 3: Amplitude versus rank, $N(n)$, plot defining cumulative distribution $P_{>}(N) \sim$ $1 / N^{\lambda}$ with the parameters of the model $\mu=1, \sigma=0.1$ and $\gamma=0.0085$. Dashed line represents asymptotic plot $N(n) \sim n^{-1 / \lambda}$ with $\lambda=3.4$.

\section{Discussion and conclusions}

We have introduced a multiplicative stochastic model for the time intervals between events of point process. Such a model of time series has only a few parameters defining the statistical properties of the system, i.e., the power-law behavior of the distribution function and the scaled power spectral density of the signal. The ability of the model to simulate $1 / f$ noise as well as to reproduce signals with the values of power spectral density slope $\beta$ between 0.5 and 1.5 promises a wide variety of applications of the model.

Let us present shortly the possible interpretations of the empirical data of the trading activity in the financial markets. With a very natural assumption of transactions in the financial markets as point events we can model the number of transactions $N_{j}$ in equal time intervals $\tau_{d}$ as the outcome of the described multiplicative point process. We already know from available studies [13] that the empirical data exhibit power spectral density in the low frequency limit with the slope $\beta \simeq 0.7$. For the pure multiplicative model $\mu=1$ this corresponds to the case when $\alpha=2 \gamma / \sigma^{2}-2 \mu \simeq-0.3$. The corresponding cumulative distribution of $N$ in the tail of high values (see equation (19)) has the exponent $\lambda=4+2 \alpha=3.4$. This is in an excellent agreement with the empirical cumulative distribution exponent 3.4 defined in [13] for 1000 stocks of the three major US stock markets.

The numerical results confirm that the multiplicative stochastic model of the time interval between trades in the financial market is able to reproduce the main statistical properties of trading activity $N$ and its power spectral density. The powerlaw exponents of the pdf of the interevent time, $\alpha$, and the cumulative distribution of the trading activity, $\lambda$, as well as the slope of power spectral density, $\beta$, are defined just by one parameter of the model $2 \gamma / \sigma^{2}$. The model suggests a simple mechanism of the power-law statistics of trading activity in the financial markets. We expect that multiplicative model of the time interval between the trades with more specific restrictions for the diffusion and more precisely adjusted parameters lies in the background of the financial markets statistics and may be useful in the financial time series analysis.

Acknowledgements

We acknowledge support by the Lithuanian State and Studies Foundation. 


\section{References}

[1] R.N. Mantegna, H.E. Stanley, Nature 376 (1995) 46.

[2] M. Levy, S. Solomon, Int.J.Mod.Phys. C 7 (1996) 595.

[3] M. Levy, S. Solomon, Physica A 242 (1997) 90.

[4] D. Sornette, R. Cont, J. Phys. I (France) 7 (1997) 431.

[5] R.N. Mantegna, H.E. Stanley, J. Stat. Phys. 89 (1997) 469.

[6] B.B. Mandelbrot, Fractals and Scaling in Finance, Springer, Berlin, 1997.

[7] P. Gopikrishnan, M. Meyer, L.A.N. Amaral, H.E. Stanley, Eur. Phys. J. B 3 (1998) 139.

[8] B.B. Mandelbrot, Physica A 263 (1999) 477.

[9] A. Blank, S. Solomon, Physica A 287 (2000) 279.

[10] P. Richmond, Eur. Phys. J. B 20 (2001) 523.

[11] J.P. Bouchaud, e-print: cond-mat/0008103, 2000.

[12] G. Bonanno, F. Lillo, R. N. Mantegna, Physica A 280 (2000) 136.

[13] P. Gopikrishnan, V. Plerou, Y. Liu, L.A.N. Amaral, X. Gabaix, H.E. Stanley, Physica A 287 (2000) 362.

[14] R.N. Mantegna, H.E. Stanley, An Introduction to Econophysics: Correlations and Complexity in Finance, Cambridge University Press, Cambridge, 2000.

[15] X. Gabaix, P. Gopikrishnan, V. Plerou, H.E. Stanley, Nature 423 (2003) 267.

[16] P. Gopikrishnan, V. Plerou, X. Gabaix, H.E. Stanley, Phys. Rev. E 62 (2000) R4493.

[17] P. Cizeau, M. Potters, J.-P. Bouchaud, Quant. Finance 1 (2001) 217.

[18] B. Kaulakys, T. Meškauskas, Phys. Rev. E 58 (1998) 7013.

[19] B. Kaulakys, Phys. Lett. A 257 (1999) 37.

[20] B. Kaulakys, T. Meškauskas, Nonlin. Anal.: Mod. Contr., Vilnius 4 (1999) 87.

[21] B. Kaulakys, Microel. Reliab. 40 (2000) 1787; cond-mat/0305067, 2003.

[22] V. Gontis, Lithuanian J. Phys. 41 (2001) 551; cond-mat/0201514, 2002.

[23] V. Gontis, Nonlin. Anal.: Mod. Contr., Vilnius 7 (2002) 43; cond-mat/0211317, 2002.

[24] F. Schmitt, D. Schertzer, S. Lovejoy, Appl. Stoch. Mod. Data Anal. 15 (1999) 29.

[25] F. Schmitt, D. Schertzer, S. Lovejoy, Int. J. Theor. Appl. Fin. 3 (2000) 361.

[26] N. Vandewalle, M. Ausloos, Eur. Phys. J. B 4 (1998) 257.

[27] P. Richmond, S. Solomon, e-print: cond-mat/0010222, 2000.

[28] B.D. Malamud, D.L. Turcotte, Stat. Planing and Inference, 80 (1999) 173.

[29] C.W. Gardiner, Handbook of Stochastic Methods, Springer, Berlin, 1986.

[30] J.-P. Bouchaud, M. Potters, Theory of Financial Risks, Cambridge University Press, Cambridge, 2000. 